\section{Rereind beti}

FEB 081991

\title{
Some Limitations of Detailed Balance for Inverse Reaction Calculations in the Astrophysical p-Process
}

D. G. Gardner

M. A. Gardner

This invited paper was presented at the Seventh International Symposium on Capture Gamma-Ray Spectroscopy and Related Topics Asilomar, Pacific Grove, California 14-19 October, 1990

December 5, 1990

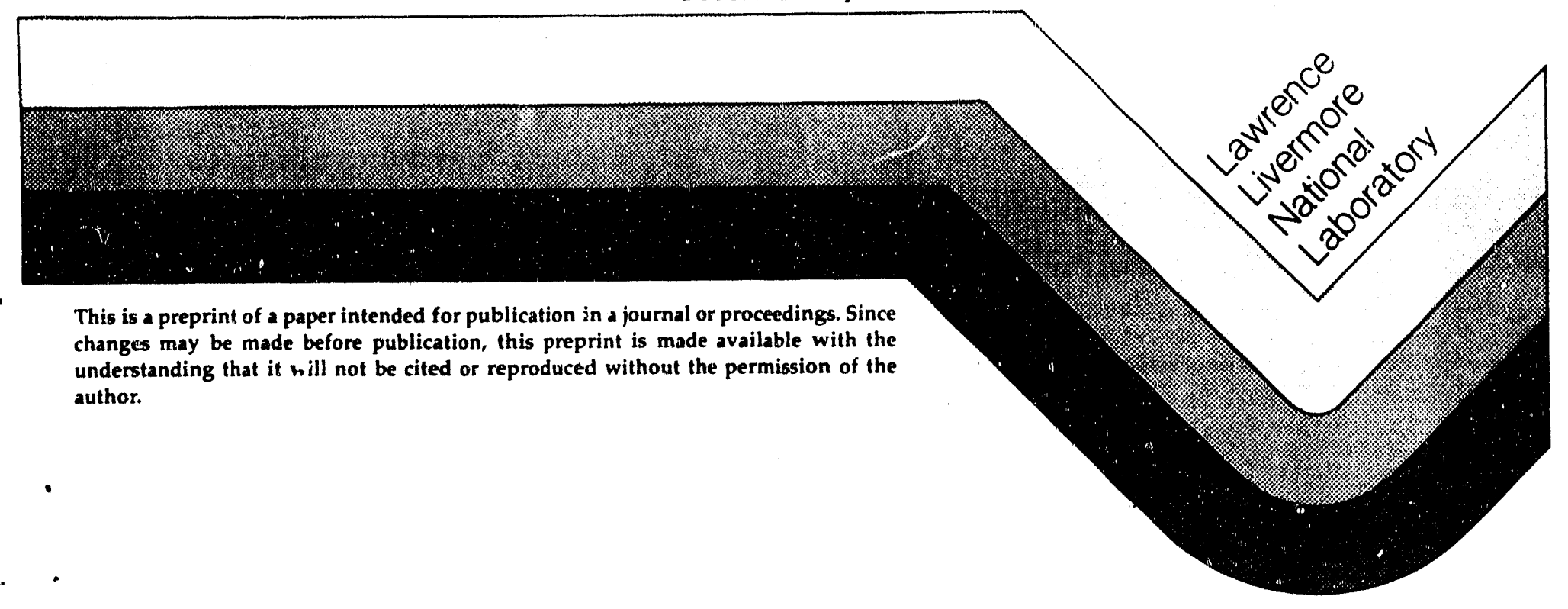




\section{DISCI.AIMER}

This document was prepared as an account of work sponsored by an agency of the United States Government. Neither the United States Government nor the University of California nor any of their employees, makes any warranty, express or implied, or assumes any legal liability or responsibility for the accuracy, completeness, or usefulness of any information, apparatus, product, or process disclosed, or represents that its use would not infringe privately owned rights. Reference herein to any specific commercial products. process, or service by trade name, trudemark, manufacturer, or otherwise, does not necessarily constitute or imply its endorsement, recommendation. or favoring by the United States Government or the University of ralifornia. The views and opinions of authors expressed herein do not necessarily state or reflect those of the United States Government or the University of California, and shall not be used for advertising or product endorsement purposes. 


\title{
SOME LIMITATIONS OF DETAILED BALANCE FOR INVERSE REACTION CALCULATIONS IN THE ASTROPHYSICAL p-PROCESS*
}

\author{
D.G. Gardner and M.A. Gardner \\ University of California, Lawrence Livermore National Laboratory \\ Livermore, CA 94551, USA
}

\begin{abstract}
p-Process modeling of some rare but stable proton-rich nuclei requires knowledge of a variety of neutron, charged particle, and photonuclear reaction rates at temperatures of 2 to $3 \times 10^{9} \mathrm{~K}$. Detailed balance is usually invoked to obtain the stellar photonuclear rates, in spite of a number of well-known constraints. In this work we attempt to calculate directly the stellar rates for $(\gamma, n)$ and $(\gamma, \alpha)$ reactions on ${ }^{151} \mathrm{Eu}$. These are compared with stellar rates obtained from detailed balance, using the same input parameters for the stellar $(n, \gamma)$ and $(\alpha, \gamma)$ reactions on ${ }^{150} \mathrm{Eu}$ and ${ }^{147} \mathrm{Pm}$, respectively. The two met' sds yielded somewhat different results, which will be discussed along with some sensitivity studies.
\end{abstract}

\section{INTRODUCTION}

The origins of the rare, proton-rich stable "p-nuclei" have been the subject of numerous investigation ${ }^{1-5}$ in which proton capture or photonuclear reactions or both have been proposed to be the important reactions involved in the p-process, the stellar mechanism for producing such nuclei. The stellar model utilizing the latter reaction type is sometimes called the $\gamma$-process. ${ }^{4}$ Such modeling requires stellar photonuclear rates which are usually obtained by detailed balance. 6 The approach is sound if the forward and reverse reactions involved proceed statistically such that, for the case $A+a=[C]=B+b$, the compound system $[C]$ has the same distributions in energy, angular momentum, and parity. However, there are a number of well-recognized constraints and in this paper we will try to determine the magnitude of the error introduced by some of these unfulfilled assumptions. We are able to attempt to analyze the validity of detailed balance for a variety of situations because our STAPLUS(Hauser-Feshbach plus spin-dependent preequilibrium) code ${ }^{7}$ allows photons in the entrance channel and also permits the spin-parity distributions in the first compound nucleus to be supplied as input.

\section{CALCULATIONAL METHOD}

The calculations presented here are for the target nucleus, ${ }^{151} \mathrm{Eu}$; the separation energies from ${ }^{151} \mathrm{Eu}$ are: $S_{n}=7.937 \mathrm{MeV}, S_{\mathrm{p}}=4.891 \mathrm{MeV}$ and $S_{\alpha}=-1.964 \mathrm{MeV}$. We have chosen europium for this effort because more experimental information exists here than for the light Gd-Sm nuclei, which are of more astrophysical interest. We wrote a code called SUNBURN to calculate photonuclear transitions from target ${ }^{151}$ Eu states available by the Boltzmann distribution at a given temperature, from some lower excitation energy (such as the ground state) up to an arbitrary maximum target excitation, the neutron separation energy for ${ }^{151}$ Eu. In most of this work, this range has been divided up into $0.1 \mathrm{MeV}$ energy bins. The photonuclear transitions

*Work performed under the auspices of the U.S. Department of Energy by the Lawrence Livermore National Laboratory under contract No. W-7405-Eng-48. 
proceed from these target bins to states above the specific reaction threshold up to another arbitrary maximum energy, which we have chosen to be $15 \mathrm{MeV}$. This is slightly above the $(\gamma, 2 n)$ threshold. Actually, we compute the photon absorption cross section, and multiply this by the branching fraction for the reaction of interest. This absorption cress section is computed from the user's choice of multipole strength functions, $f_{x l}\left(E_{\gamma}\right)$, and so is used with each target-state spin, $J\left(E_{l}\right)$, to produce the spin and parity distribution in the ${ }^{151} \mathrm{Eu}$ compound nucleus:

$$
\sigma_{\gamma \mathrm{ab}}^{\mathrm{J} \pi}\left(\mathrm{E}_{\gamma}\right)=3.85 \times 10^{3}\left(\frac{2 \mathrm{~J}+1}{2 \mathrm{~J}\left(\mathrm{E}_{\mathrm{t}}\right)+1}\right) \sum_{\mathrm{x} l} \mathrm{E}_{\gamma}{ }^{2 l-1} \mathrm{f}_{\mathrm{x} l}\left(\mathrm{E}_{\gamma}\right) \text { barn. }
$$

Multiplying this by the appropriate energy-, spin-, and parity-dependent branching fraction greatly simplifies the programming, and allows easy problem modifications.

\section{STELLAR PHOTO-REACTION RATE}

Assume Boltzmann-type thermal equilibrium

$$
\begin{aligned}
& \lambda_{\gamma x}=\operatorname{CON} /\left(2 J_{0}+1\right) * \int_{E_{1}}^{E_{a}} d E_{t} * \frac{\rho\left(E_{t}\right) * B F\left(E_{t}\right) *\left(2 J\left(E_{t}\right)+1\right)}{Q /\left(2 J_{0}+1\right)} \int_{\substack{E_{z} \\
z 0}}^{E_{h}-E_{t}} d E_{\gamma_{t}} * \operatorname{PLNK}\left(E_{\gamma}\right) * \sigma_{\gamma a b}\left(E_{\gamma}\right) * \\
& Q=\sum \rho\left(E_{t}\right) * B F\left(E_{t}\right) *\left(2 J\left(E_{t}\right)+1\right) \\
& \rho\left(E_{t}\right)=\text { target level density } \mathrm{MeV}^{-1} \\
& \operatorname{CON}=2.9979 \mathrm{e}+10 * 1 . \mathrm{e}-24 \mathrm{~cm}^{3} \mathrm{sec}^{-1} \mathrm{~b}^{-1} \\
& J\left(E_{t}\right)=\text { most probable } J \text { at target } E_{t} \\
& B F\left(E_{t}\right)=\text { Boltzmann factor at } E_{t} \\
& \operatorname{PLNK}\left(\mathrm{E}_{\gamma}\right)=\text { Planck spectrum photons } \mathrm{cm}^{-3} \mathrm{MeV}^{-1} \\
& \sigma_{\text {yab }}=\text { photon absorption cross section } b \\
& \sigma_{\gamma \mathbf{x}}(E)=(\gamma, x) \text { cross section at } E=E_{\gamma}+E_{t} \\
& E_{t} \text { is target energy where photon transitions start } \\
& E_{l} \text { is lowest target energy (MeV) } \\
& E_{\mathrm{h}} \text { is highest target energy }(\mathrm{MeV}) \\
& E_{\mathbf{x}} \text { is }(\gamma, x) \text { threshold }(\mathrm{MeV}) \quad E_{\mathbf{s}}=\text { neutron separation energy }(\mathrm{MeV}) \\
& \left(\frac{\sigma_{\gamma x}\left(E_{\gamma}+E_{t}\right)}{\sigma_{\gamma a b}\left(E_{\gamma}\right)}\right) \sec ^{-1}
\end{aligned}
$$

Fig. 1. Equation for computing stellar photonuclear reaction rates that is incorporated in the computer code SUNBURN.

The stellar photonuclear rate expression is given in Fig. 1. All calculations were performed on an energy grid with $0.1 \mathrm{MeV}$ steps. The excitation functions $\sigma_{\mathrm{pab}}\left(\mathrm{E}_{\gamma}\right)$ and $\sigma_{\gamma x}(\mathrm{E})$ were calculated with the Hauser-Feshbach plus precompound evaporation code STAPLUS, ${ }^{7}$ and interpolated onto the energy grid using a piece-wise cubic Hermite polynomial routine; 8 the integrations were done using the trapezoidal rule. The integration accuracy was checked at $T 9=2$ by integrating the product of the level density and the Boltzmann factor, from 0 to $8 \mathrm{MeV}$, and comparing the answer with that obtained in closed form. The agre ment was better than $1 \%$, which may not be sufficiently accurate. We hope to try a Gaussian quadrature method in the future. 
The neutron transmission coefficients were obtained using the coupled channel code ECIS 9 and a deformed optical model potential. ${ }^{10}$ The proton ${ }^{11}$ and alpha particle ${ }^{12}$ potentials were both spherical. The alpha particles presented the greatest problem when computing transmission coefficients. None of our spherical or deformed potential codes were accurate for transmission coefficients below about $10^{-15}$ in magnitude. We intend to modify our codes to incorporate double-precision routines that compute Coulomb wave functions with greatly increased accuracy. In the present work, we graphically extended both $\sigma(\alpha, \gamma)$ and $\sigma(\gamma, \alpha)$ by the barrier penetration method from $\mathrm{E}_{\alpha}=6$ down to about $4.5 \mathrm{MeV}$. This will not affect our results at $T_{9}=3$, but might at $T_{9}=2$. The multipole strength functions we favor for dipole and isoscalar E2 transitions have been described elsewhere. 13 Our unadjusted strength functions produce photoneutron reactions on ${ }^{151} \mathrm{Eu}$ and ${ }^{153} \mathrm{Eu}$ that compare well with the experimental data. ${ }^{14}$ Our unadjusted calculations agree very well with the ENDF/B evaluations for $(n, 2 n)$ and $(n, 3 n)$ reactions and with the neutron capture data ${ }^{10}$ for the $(n, \gamma)$ reactions on targets of both ${ }^{151} \mathrm{Eu}$ and ${ }^{153} \mathrm{Eu}$. Testing the choice of alpha particle optical potentials is quite important; using the Igo volume potential ${ }^{15}$ yields a $(\gamma, \alpha)$ excitation function that is between 3 and 6 times the magnitude generated using the McFadden surface derivative potential ${ }^{12}$. We compared our calculations with data ${ }^{16}$ for the $(\alpha, n)$ and $(\alpha, \gamma)$ reactions on ${ }^{139} \mathrm{La}$ and found the results to be quite satisfactory.

In order to compare our calculated stellar photonuclear reaction rates or rate factors with those computed via detailed balance, we follow the usual treatment ${ }^{6}$ and calculate the capture cross sections using the discrete target levels (14 to 32) weighted in their true sequence by the Maxwell-Boltzmann and $(2 \mathrm{~J}+1)$ factors and the partition funstion. In the continuum we use a representative capture cross section derived from the above weighting sequence. In standard notation, the detailed balance expression for the stellar photonuclear reaction rate factor $N_{\Lambda}<\bar{\sigma}_{\gamma \mathrm{x}} \mathrm{v}>*$ is related to the stellar rate factor for the $(x, \gamma)$ capture reaction, $N_{A}<\bar{\sigma}_{x} \gamma^{v}>$, by

$$
N_{A}<\bar{\sigma}_{\gamma x} v>*=\left(\frac{g_{I} g_{X}}{g_{L}}\right)\left(\frac{Q_{I}}{Q_{L}}\right)\left(\frac{A_{I} A_{x}}{A_{L}}\right)^{3 / 2}\left(\frac{k T}{2 \pi \hbar^{2} N_{A}}\right)^{3 / 2} N_{A}<\bar{\sigma}_{x \gamma} v>* \exp \left[-\left(S_{X}+E_{x}\right) / k T\right] .
$$

We have changed the symbol for the partition function from $G$ to $Q$, and that for the $Q$-value to $S_{x}+E_{x}$ to conform with Fig. 1. Here, $S_{x}$ is the separation energy of particle $X$ from the compound nucleus (say, ${ }^{151} \mathrm{Eu}$ ) and $E_{X}$ is that particle's incident energy in the center of mass system. Note that the stellar photonuclear rate factor is equal to the actual stellar rate for nuclei at thermal equilibrium exposed to a Planck photon distribution, i.e., the rate $\lambda_{y x} \sec ^{-1}$ in Fig. 1 is numerically equal to the rate factor $N_{A}<\bar{\sigma}_{\gamma x} v>*$.

We may see that the two rate factors in Eq. 2 are linearly proportional; if Igo's transmission coefficients had been used instead of McFadden's, the stellar photonuclear reaction rate would have been a factor of 3 to 6 times faster than the rate we calculate. Of particular interest is the effect produced by low-energy charged particles trying to penetrate the Coulomb barrier in the $(x, \gamma)$ reaction. For example, alpha particles on ${ }^{147} \mathrm{Pm}$, producing the compound system ${ }^{151} \mathrm{Eu}$, are extremely insensitive to the photon widths, $\Gamma_{\gamma}\left(E_{\gamma}\right)$, and therefore to either the photon transmission coefficients or the level density in ${ }^{151} \mathrm{Eu}$. Either of the two may be divided by one or more orders of magnitude without changing the $\sigma(\alpha, \gamma)$ cross section, because of the very small size of $\Gamma_{\alpha}\left(E_{\alpha}\right)$. Below the neutron separation energy in ${ }^{151} \mathrm{Eu}$, the $\sigma(\alpha, \gamma)$ cross section is only the alpha particle reaction cross 
section. There is essentially no information concerning photons contained within $\mathrm{N}_{\mathrm{A}}<\bar{\sigma}_{\alpha \gamma} \nu>^{*}$, and yet Eq. 2 predicts a stellar $(\gamma, \alpha)$ reaction rate! The explanation, of course, is that Eq. 2 predicts one number, for a given temperature, that is the convolution of the Planck spectrum with some photonuclear excitation function, properly weighted and summed over all available target states. There are an infinite number of multipole strength-function combinations that will yield the same numerical rate, even if concurrent and complimentary variations in the ${ }^{151}$ Eu level density are ignored.

One final comment should be made concerning Eq. 2. Assume for a moment that only dipole transitions are important for photonuclear reactions. These will produce a very narrow compound nucleus spin and parity distribution, as compared with that produced by incident particles, particularly the large number of partial waves brought in by alpha particles. To make the comparison of the rates calculated by the equation in Fig. 1 with that derived from detailed balance, we have utilized the feature of the STAPLUS code to read as input the normalized spin and parity distributions that a particle would produce in the compound nucleus and to distribute the photon absorption cross section accordingly, before allowing the photonuclear reactions to proceed.

\section{RESULTS}

In Fig. 2a, we show the calculated excitation function for ${ }^{147} \mathrm{Pm}(\alpha, \gamma)^{151} \mathrm{Eu}$, with photon transmission coefficients from our systematics, and with these decreased by a factor of 10 . Below the neutron separation energy in ${ }^{151} \mathrm{Eu}$ there is no visible difference between the two calculations. The same effect is found when the ${ }^{151} \mathrm{Eu}$ level density is decreased by the same amount. As mentioned in the previous section, the alpha particle is primarily exploring the Coulomb barrier, while the exponentially decreasing tail of the nuclear wave function interacts only slightly with the nucleus.
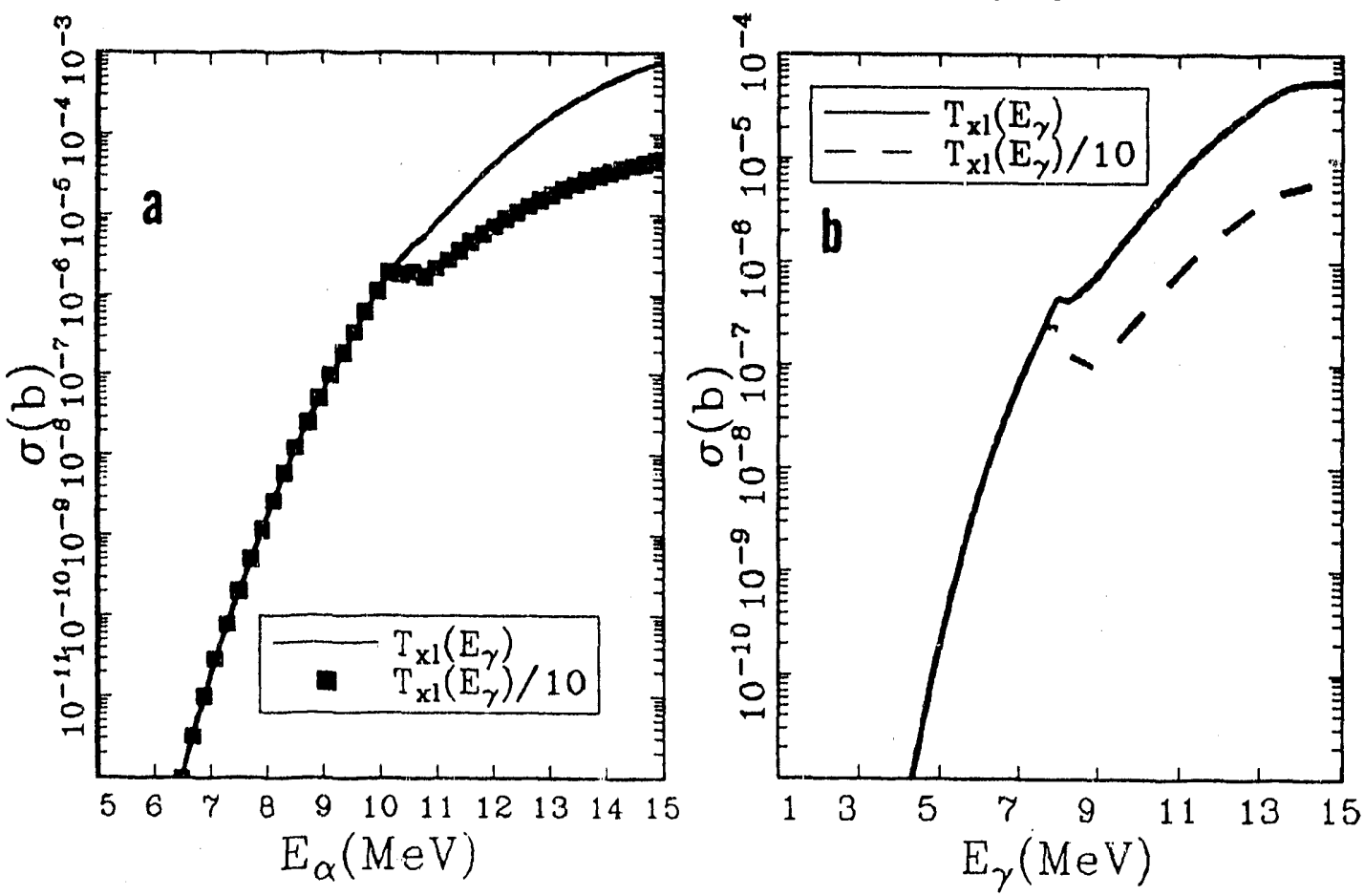

Figs. 2a,b. Change in the ${ }^{147} \mathrm{Pm}(\alpha, \gamma)^{151} \mathrm{Eu}$ and ${ }^{151} \mathrm{Eu}(\gamma, \alpha)^{147} \mathrm{Pm}$ cross sections due to $1 / 10$ decrease in photon transmission coefficients. 
Suppressing spin and parity,

$\sigma_{\alpha \gamma}\left(E_{\alpha}\right) \approx \pi \star^{2}\left(\frac{2 \pi \Gamma_{\alpha O}}{D}\right)\left(\frac{\Gamma_{\gamma}}{\Gamma_{\gamma}+\Gamma_{\alpha}+\Gamma_{n}}\right)$

Recall that at excitation energies in ${ }^{151} \mathrm{Eu}$ below the neutron separation energy, $\sigma_{\alpha \gamma}\left(E_{\alpha}\right)$ is merely the alpha particle reaction cross section, $\sigma_{\alpha \mathrm{Rx}}\left(\mathrm{E}_{\alpha}\right)$. The symbol $\Gamma_{\alpha_{0}}$ represents the transition from the ground state of ${ }^{147} \mathrm{Pm}$ to the continuum in ${ }^{151} \mathrm{Eu}$, and we have observed that $\Gamma_{\alpha_{0}}$ is usually $\geq 90 \%$ of the total width, $\Gamma_{\alpha}$. Because $\Gamma_{\gamma}$ is many orders of magnitude larger than $\Gamma_{\alpha}$, very large changes in $\Gamma_{\gamma}$ produce no noticeable effect. In Fig. $2 b$, the reverse reaction is shown. Following the above argument concerning the relative sizes of the widths $\Gamma_{\alpha}$ and $\Gamma_{\gamma}$ it is easy to show why changing $\Gamma_{\gamma}$ when $\Gamma_{n}$ is zero produces no effect, but at higher excitation energies in ${ }^{151} \mathrm{Eu}$, accessed by photons, a decrease in their transmission coefficients by a factor of $1 / 10$ results in essentially the same decrease in $\sigma(\gamma, \alpha)$. For temperatures in the range of $\mathrm{T} 9=2$ or 3 , the Planck photon distribution convolutes primarily with the low-energy portion of the $(\gamma, \alpha)$ excitation function, and detailed balance calculations should be impacted only slightly. At higher temperatures a correct representation of the multipole strength becomes steadily more important, until such high temperatures are reached that multiple-particle emission occurs, such as $(\gamma, 2 n)$ or $(\gamma, \alpha n)$, at which point the detailed balance approach is no longer useful. The approach of calculating directly the photonuclear reactions and their stellar rates, does not suffer from these restrictions, and clearly is useful in signaling the energy threshold where some of the restrictions of detailed balance begin to appear and hence the temperature range where detailed balance becomes less accurate.

Perhaps a more interesting illustration of the fact that detailed balance provides only a minimal constraint on the multipole strength functions, $f_{x l}\left(E_{\gamma}\right)$, involves a comparison of the results using a giant dipole resonance shape for E1 strengths with the energy-independent $f_{E} 1$ from the Weisskopf single-particle approach. We applied a hindrance factor of $1 \times 10^{-3}$ to the Weisskopf $f_{E 1}$ and normalized the $f_{E 1}+f_{M 1}$ such that $2 \pi \Gamma \gamma / D=3.95$ at the neutron separation energy in ${ }^{151} \mathrm{Eu}$ - the same value our dipole systematics produced. Figs. $3 \mathrm{a}$ and $3 \mathrm{~b}$ show the anticipated effects on the $(\gamma, \alpha)$ reaction of the two vastly different functional forms for $f_{E 1}$, even when both $\Gamma_{\gamma}$ 's were set equal at $S_{n}$. The effect on $\sigma(\alpha, \gamma)$ is barely discernable. This complete dominance by the Coulomb barrier may also be observed in the calculation of $\left.\mathrm{N}_{\mathrm{A}}<\bar{\sigma}_{\not \gamma \gamma}\right\rangle^{*}$, the forward rate factor in the detailed balance equation shown in Eq. 2. For ${ }^{147} \mathrm{Pm}$, we used 14 discrete levels up to $0.807 \mathrm{MeV}$. Figure $4 \mathrm{a}$ presents a selection of ${ }^{147} \mathrm{Pm}(\alpha, \gamma){ }^{151} \mathrm{Eu}$ excitation functions. For target spins of 0.5 to 5.5 , and for level energies from zero to $0.807 \mathrm{MeV}$, the excitation functions overlap so well that a single curve was used to represent the collection in the convolution with the Maxwell-Boltzmann function. The order of the spin sequence in the $(2 \mathrm{~J}+1)$ weighting was immaterial. This was not true in the $(n, \gamma)$ case, as may be seen in Fig. 4b. Because of the Boltzmann factor weighting of the $(2 \mathrm{~J}+1)$ term, the order of the spin sequence with excitation energy should not be neglected; the MaxwellBoltzmann averaged capture cross section from each of the 27 levels in ${ }^{150} \mathrm{Eu}$ varied significantly.

The insensitivity of the stellar $(\gamma, \alpha)$ rate, below the $(\gamma, n)$ threshold, to variations in the multipole strength-function modeling is not observed in the stellar $(\gamma, n)$ rate because $\Gamma_{\gamma}$ cannot be neglected as compared with $\Gamma_{n}$ until the ${ }^{15 i}$ Eu nucleus is several $\mathrm{MeV}$ above the $(\gamma, n)$ threshold. Figure 5 displays the effect produced in the calculated ${ }^{151} \mathrm{Eu}(\gamma, \mathrm{n}){ }^{150} \mathrm{Eu}$ reaction by a $1 / 10$ decrease in the photon transmission 

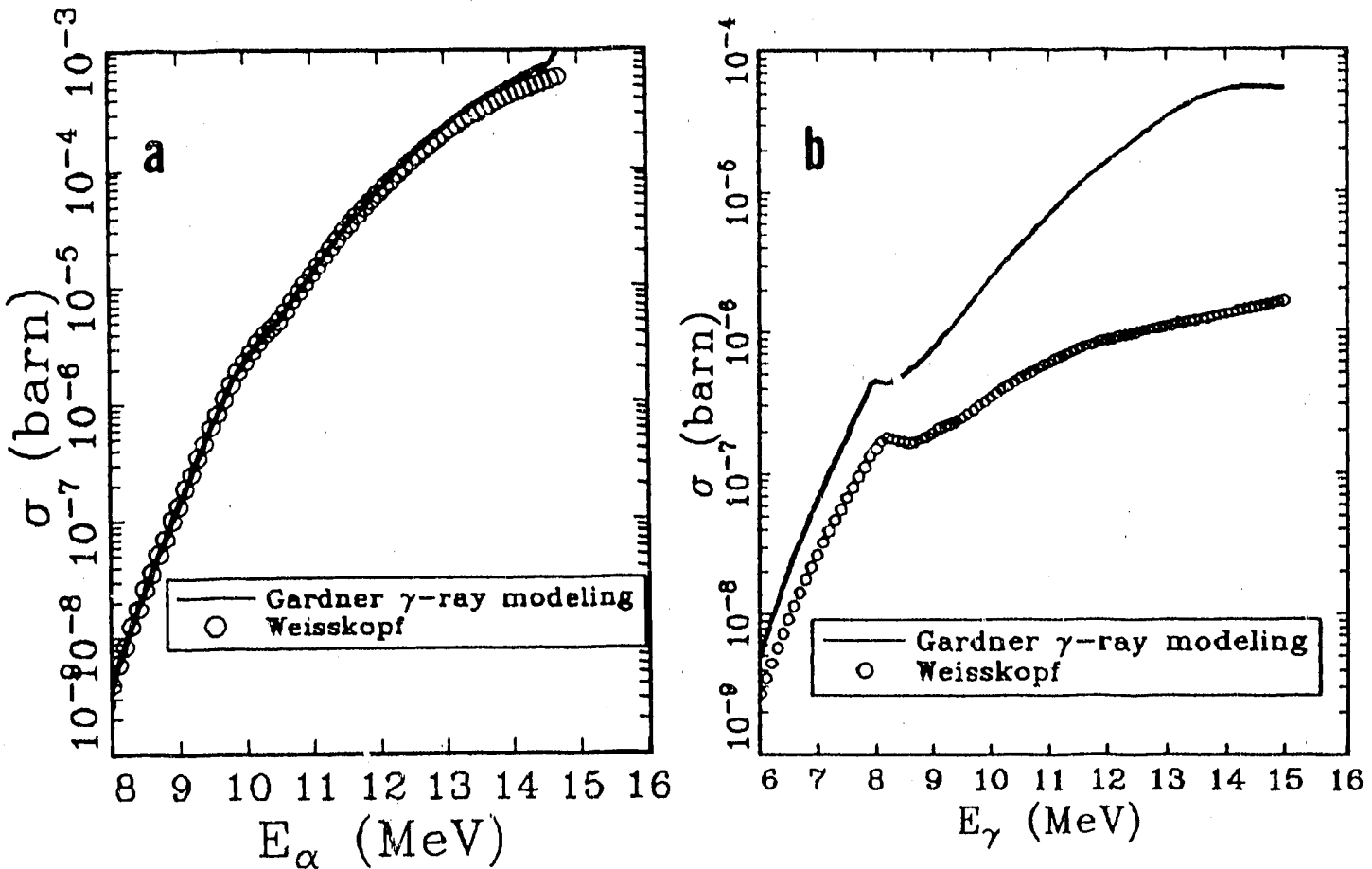

Figs. 3a,b. Change in the ${ }^{147} \mathrm{Pm}(\alpha, \gamma){ }^{151} \mathrm{Eu}$ and ${ }^{151} \mathrm{Eu}(\gamma, \alpha)^{147} \mathrm{Pm}$ cross sections due to different dipole strength functions.
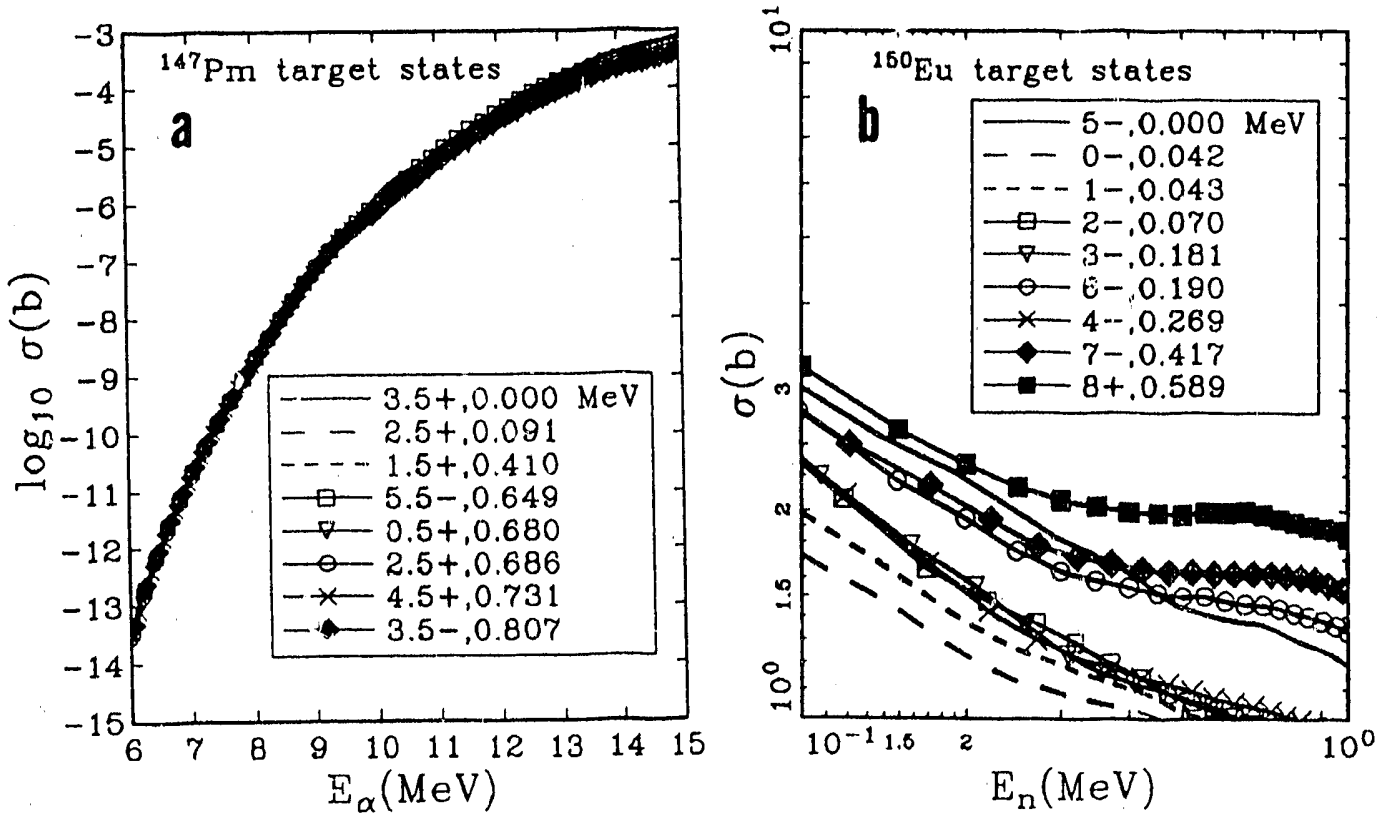

Figs. 4a,b. ${ }^{147} \mathrm{Pm}(\alpha, \gamma){ }^{151} \mathrm{Eu}$ and ${ }^{150} \mathrm{Eu}(n, \gamma){ }^{151} \mathrm{Eu}$ excitation functions for a selection of discrete level target states. 


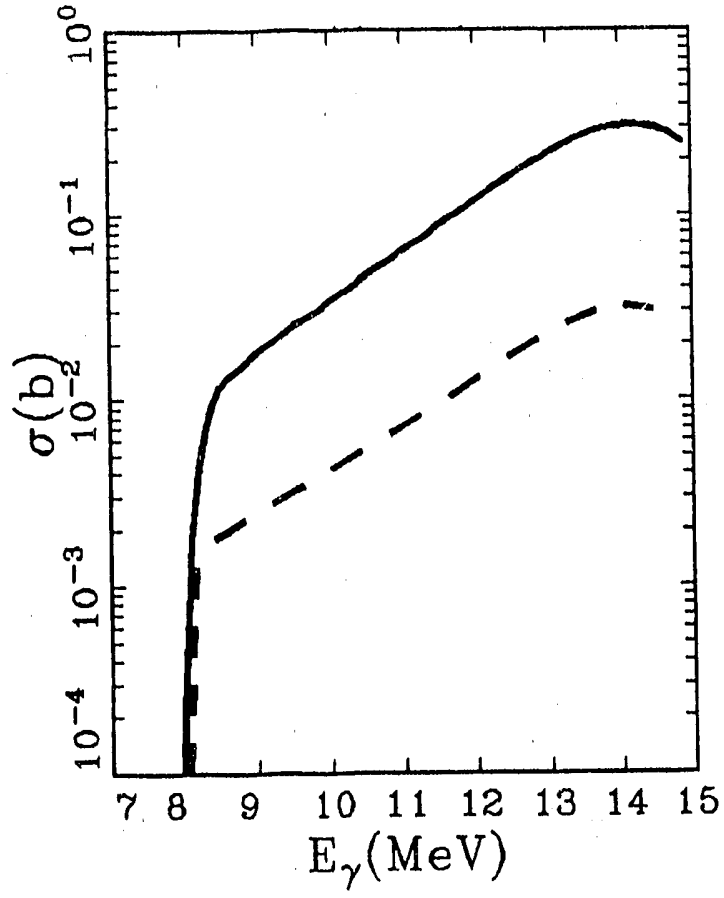

Fig. 5. Change in ${ }^{151} \mathrm{Eu}(\gamma, \mathrm{n})^{150} \mathrm{Eu}$ cross section due to $1 / 10$ decrease (--) in multipole strength functions. coefficients; the decrease in $\sigma(\gamma, n)$ is also about $1 / 10$, while the $(n, \gamma)$ capture cross section decreases only about $40 \%$. The stellar photoneutron rates show a factor-of-two decrease for $T_{9=2}$ to 3 . Because the Planck distribution decreased by about $10^{5}$ at $\mathrm{T}_{9}=2$, for ${ }^{151} \mathrm{Eu}$ excitation energies of 8 to $1 \mathrm{uMeV}$, we are not sure that our integration of the equation in Fig. 1 in steps of 0.1 $\mathrm{MeV}$ is sufficiently accurate. From 8.5 to $9.5 \mathrm{MeV}$ of excitation, for example, the branching fraction $\sigma(\gamma, n) / \sigma_{\gamma a b}$ only changes from 0.59 to 0.77 , but is 0.014 at $8.0 \mathrm{MeV}$. Not allowing $\gamma$-ray competition in the first compound nucleus increases the stellar photoneutron rate by almost a factor of 5 . We plan to study other integration methods in the near future.

In Figs. $6 a$ and $6 b$, we show our calculated stellar rates for the $(\gamma, n)$ and $(\gamma, \alpha)$ reactions on ${ }^{151} \mathrm{Eu}$, for temperatures of $\mathrm{T}_{9}=2$ to 3 . The curves show the rates vs target excitation energy, after which the total integrated rate is given. The Gamov peak values for the $(\alpha, \gamma)$ reaction at iemperatures of $T_{9}=2.0,2.5$, and 3.0 are, respectively, $5.5,6.9$, and $8 \mathrm{MeV}$; for the corresponding $\mathrm{E}_{\alpha}$ energies add $2 \mathrm{MeV}$. These peak values are reasonable for the convolution of the Maxwell-Boltzmann function with the $(\alpha, \gamma)$ excitation function, whereas for the stellar $(\gamma, \alpha)$ rate functions shown in Fig. 6a the skewing of the curves' peak values to much lower excitation energies emphasizes the dramatic effect due to the shape of the Planck photon distribution. Hence, very low energy alpha particles contribute greatly to the stellar photonuclear rates. In Fig. $6 \mathrm{~b}$, our arbitrary cut off of ${ }^{151} \mathrm{Eu}$ target states at $8 \mathrm{MeV}$ might appear to be too low an energy. However, an examination of the curve values near $8 \mathrm{MeV}$ indicates little of the total integral was lost. Also, the behavior of the curves at low energies shows that discrete level information for the target nucleus is essentially not needed due to the moderation of the Boltzmann facior by the rapidly increasing level density function. Should one wish to compute stellar photonuclear rates at even higher temperatures, rate curves such as these, along with the calculated values of the neutron widths, should help decide the highest energy in ${ }^{151} \mathrm{Eu}$ where states are sufficiently long lived to be considered available target states.

We now wish to compare our stellar photonuclear reaction rates calculated directly with those obtained via detailed balance. First we must discuss the spinparity distribution in the first compound nucleus, which must be the same for the forward and reverse reactions if detailed balance is to obtain. Photons produce a narrow distribution regardless of photon energy; an extreme example would be dipole transitions from a spin-zero target--only $\mathrm{J}=1$ states could be produced. Neutrons incident on the $(\mathrm{Z}, \mathrm{N}-1)$ nucleus can produce a much broader distribution, even for relatively low incident energies. Alpha particles can bring in many partial waves and 

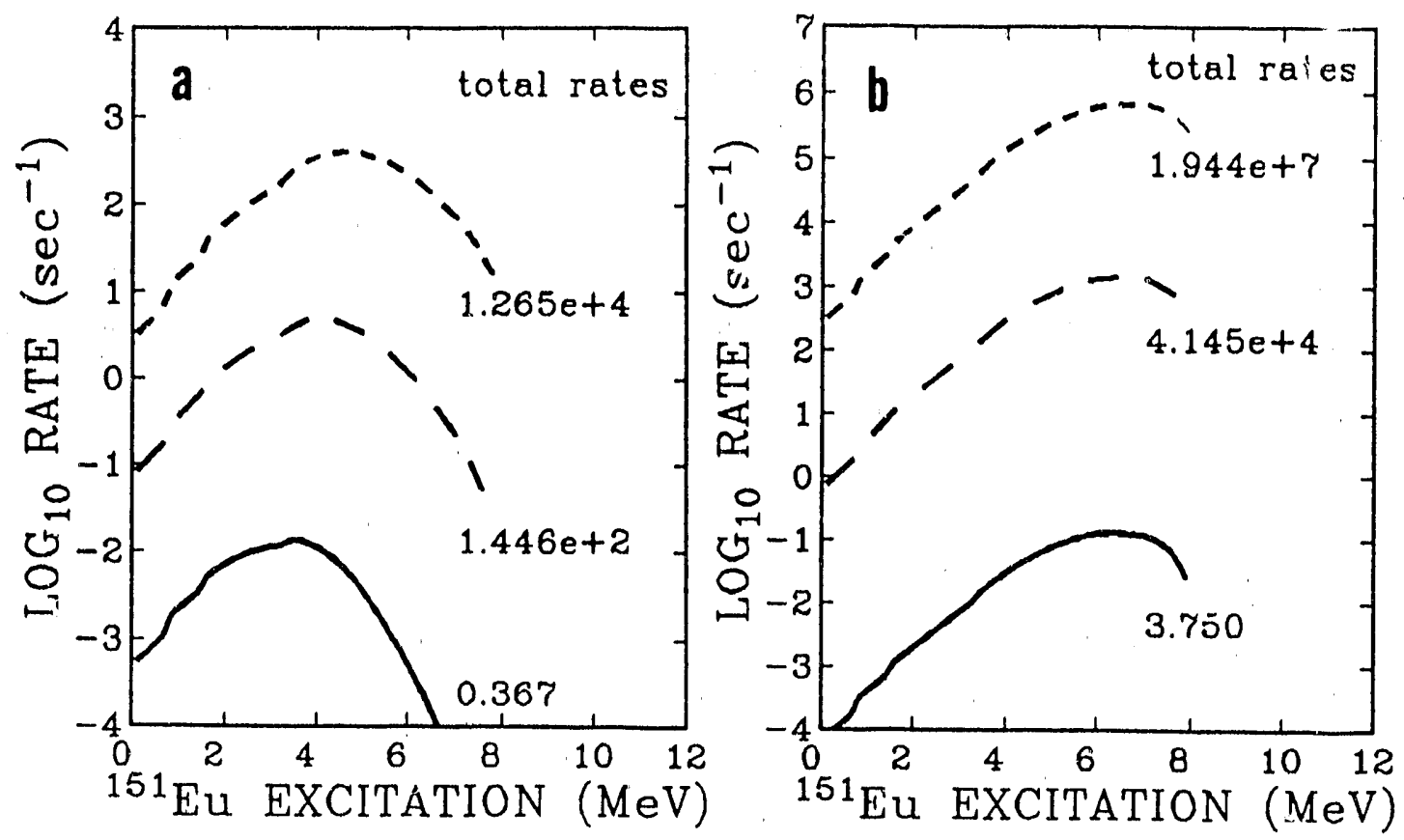

Figs. 6a,b. Stellar photonuclear rates vs target excitation energy for the $(\gamma, \alpha)^{147} \mathrm{Pm}$ and $(\gamma, n)^{150} \mathrm{Eu}$ reactions, respectively, plus total rates.

produce very broad distributions. As noted above, our STAPLUS code allows us to supply a spin and parity distribution for the first compound nucleus, normalized to the actual (photon) reaction cross section. We then repeared our $(\gamma, n)$ and $(\gamma, \alpha)$ excitation function calculations, changing the initial spin distribution to that which would have been produced by a neutron or an alpha particle of the appropriate centerof-mass energy, for ${ }^{151} \mathrm{Eu}$ excitation energies of $15 \mathrm{MeV}$ down to the reaction threshold. The results appear in Figs. $7 \mathrm{a}$ and $7 \mathrm{~b}$, where the ratio of the directly calculated stellar rates to those obtained via detailed balance are given for the two types of photonuclear calculations--the normal calculation using the photon-produced spin distribution and the modified calculation using the particle-produced distribution as input. For the first case, both the $(\gamma, \alpha)$ and $(\gamma, n)$ rates are about twice those inferred from detailed balance. When the particle-produced distributions were used, the $(\gamma, \alpha)$ ratios differed not greatly from unity, whereas the $(\gamma, n)$ ratios decreased about $20 \%$ or so.

From our results so far, it cannot be said unequivocally that the direct calculation of stellar photonuclear rates is a more accurate procedure than the traditional detailed balauce approach. However, because both the $(\gamma, \alpha)$ and $(\gamma, n)$ stellar rates differ from the detailed balance results by about the same factor, and the reactions are so vastly different, there is some suggestion that the direct calculational approach is more reliable. Recall the function $J\left(E_{j}\right)$ in the equation in Fig. 1. For ${ }^{151} \mathrm{Eu}$ this function ranges from 1.5 at low target excitation energies to 5.5 for target energies near 8 $\mathrm{MeV}$. As such, it tends to resemble the neutron distribution (although the higher spins are not represented), but it does not emulate the alpha particle distribution. Therefore, when the latter distributions are inserted into the $(\gamma, \alpha)$ calculations, Fig. $7 \mathrm{a}$ shows that the results reproduce those from detailed balance. Because the nentron distributions are more accurately reproduced by the $J\left(E_{1}\right)$ function, in the direct stellar rate calculation, their insertion as input produces only the small change shown in Fig. $7 \mathrm{~b}$. This suggests to us that the detailed balance requirement of identical 
spin-parity distributions for the forward and reverse reactions is not being fulfilled-certainly not in our calculations and probably not in nature.
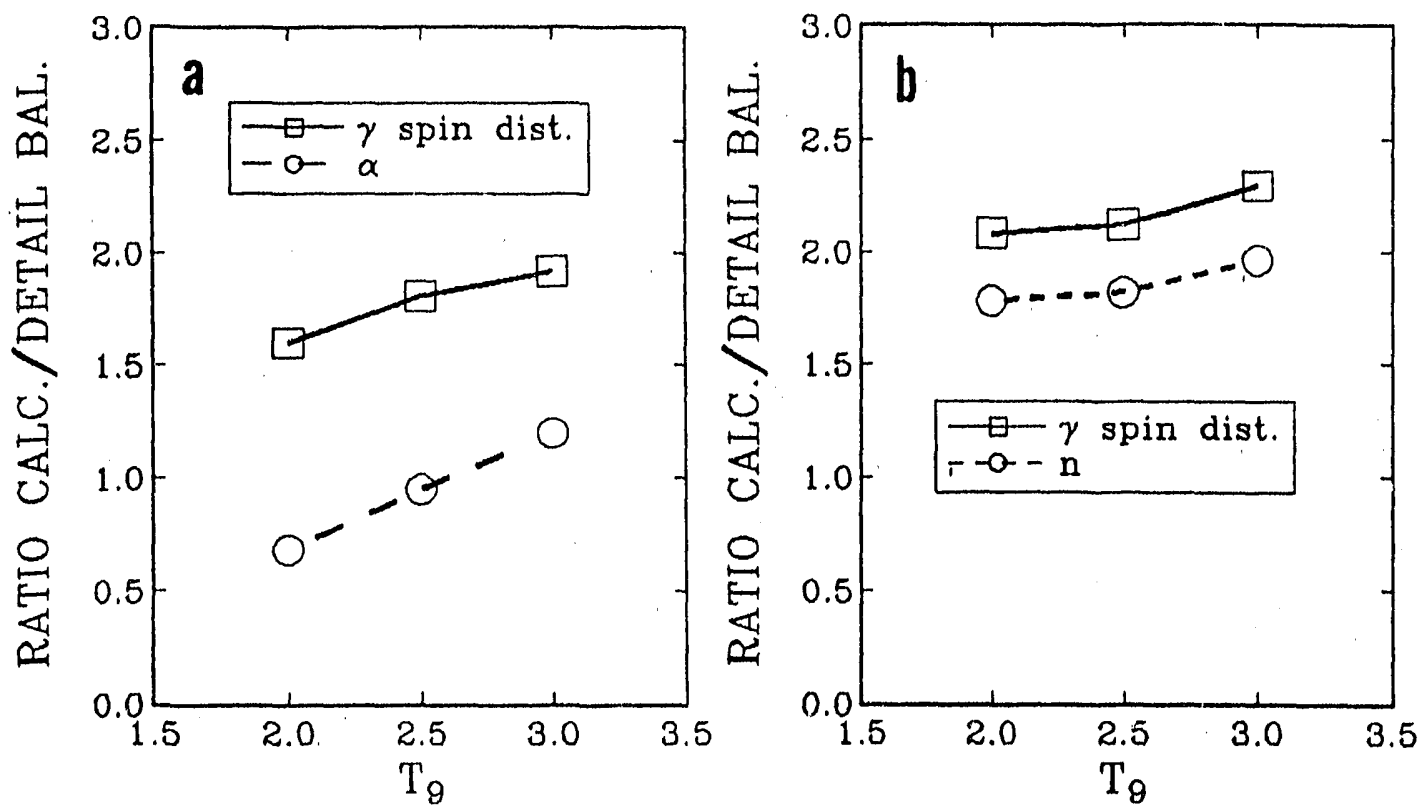

Figs. 7a,b. Stellar rates for ${ }^{151} \mathrm{Eu}(\gamma, \alpha)^{147} \mathrm{Pm}$ and ${ }^{151} \mathrm{Eu}(\gamma, \mathrm{n}){ }^{150} \mathrm{Eu}$ reactions obtained from direct calculations ratioed to those inferred from detailed balance.

\section{CONCLUSIONS}

Using ${ }^{151} \mathrm{Eu}$ as the target isotope and with realistic nuclear parameters, we have compared the direct calculations of the stellar photo-reaction rates with those inferred from detailed balance. Because interest in the astrophysical p-process initiated this study, the temperature range was confined to $T_{9}=2$ to 3 . A number of conclusions may be drawn:

1. The directly computed stellar photonuclear rates for the $(\gamma, \alpha)$ and $(\gamma, n)$ reactions were about 2 to 3 times the rates inferred from detailed balance. It is argued, but not proved, that the direct calculational approach is the more accurate.

2. The direct computational approach is not limited to a single emitted particle; multiple particle or no particle emission may be treated as easily, i.e. $(\gamma, 2 n)$, $(\gamma, \alpha n)$, a ground state populating an isomer, an isomeric state depopulated back to the ground state, etc.

3. The direct calculation is much less dependent on knowledge of the low-energy nuclear level structure than the usual approach.

4. The photonuclear excitation functions themselves provide useful information concerning the temperature at which the inverse reaction may begin to deviate from the restrictions imposed by detailed balance. Further, when they are used to produce stellar photonuclear rates as a function of the target excitation energy, one obtains additional information on the best choice of the energy range of the integrals shown in Fig. 1.

5. The p-process nuclei are stable while their predecessors often are not. Photonuclear reactions on stable nuclei should be easier to compute than capture reactions on unstable nuclei, simply because more experimental data are available to supply input to the nuclear reaction calculations. 


\section{ACKNOWLEDGMENTS}

The author: are indebted to S.E. Woosley and R.A. Ward for valuable conversations that greatly increased our understanding of the astrophysical aspects of this work, and to W.M. Howard who first introduced us to the pprocess and some of the problems involved in the relevant rate factor calculations. Special thanks are given to Sue Frumenti for the preparation of this manuscript.

\section{REFERENCES}

1. E.M. Burbidge, G.R. Burbidge, W.A. Fowler, and F. Hoyle, Rev. Mod. Phys. 29, 547 (1957).

2. J. Audouze and J.W. Truran, Astrophys. J. 202, 204 (1975).

3. M. Arnould, Astron. Astrophys. 46. 117 (1976).

4. S.E. Woosley and W.M. Howard, Ap. J. Suppl. 36, 285 (1978).

5. M. Rayet, N. Prantzos, and M. Arnould, Astron. Astrophys. 227, 271 (1990).

6. J.A. Holmes, S.E. Woosley, W.A. Fowler, and B.A. Zimmerman, Atomic Data and Nuclear Data Tables 18, 305 (1976) and references therein.

7. Our version of the STAPRE code originally written by $M$. Uhl and $B$. Strohmaier, Report IRK 76/01 (1976, updated 1978).

8. F.N. Fritsch, Lawerence Livermore National Laboratory Report, UCID30194 (1982).

9. J. Raynal, Report IAEA-SMR-9/8 (1972).

10. R.L. Macklin and P.G. Young, Nucl. Sci. Eng. 95,189 (1987).

11. C.M. Perey and F.G. Perey, Atomic Data and Nuclear Data Tables 17, 6 (1976).

12. L. McFadden and G.R. Satchler, Nucl. Phys. 84, 177 (1966).

13. D.G. Gardner, Chapter III, Neutron Radiative Capture, Vol. 3, ed. R. Chrien (Pergamon Press, Oxford, 1984), p. 91-101; one free parameter, $E_{\mathbf{x}}$, changed to $11 \mathrm{MeV}(1984)$.

14. T.J. Boal, E.G. Muirhead, and D.J.S. Findlay, Nucl. Phys. A406, 257 (1983).

15. J.R. Huizenga and G.J. Igo, Argonne National Laboratory Report ANL-6373 (1961).

16. E.V. Verdieck and J.M. Miller, Phys. Rev. 153, 1253 (1967). 
